Enfermagem Brasil 2018;17(3):208-17

\title{
ARTIGO ORIGINAL \\ Segurança do paciente: desenvolvimento do tema em cursos de graduação em enfermagem
}

Mariana Neves de Araújo Lopes, M.Sc.*, Lúcia Marinilza Beccaria, D.Sc.**, Lidia Beloni Silva, M.Sc. ${ }^{* \star}$, Claudia Bernardi Cesarino, D.Sc. ${ }^{\star * \star}$, Josimerci Ittavo Lamana Faria*****

*Enfermeira, Programa de Pós Graduação em Enfermagem da Faculdade de Medicina de São José do Rio Preto, ${ }^{* *}$ Prof ${ }^{a}$ do Departamento de Enfermagem Especializada da Faculdade de Medicina de São José do Rio Preto, ${ }^{* * *}$ Enfermeira, Programa de Pós Graduação em Enfermagem da Faculdade de Medicina de São José do Rio Preto, ${ }^{* * * *}$ Prof ${ }^{a}$ do Departamento de Enfermagem Geral da Faculdade de Medicina de São José do Rio Preto, ${ }^{* * * * *}$ Prof $^{a}$ do Departamento de Enfermagem Especializada da Faculdade de Medicina de São José do Rio Preto

Recebido em 4 de agosto de 2017; aceito em 19 de março de 2018.

Endereço para correspondência: Lúcia Marinilza Beccaria, Av. Francisco das Chagas Oliveira, 2550 casa 41 Higienópolis 15085-485 São José do Rio Preto SP, E-mail: lucia@famerp.br; Mariana Neves de Araújo Lopes: mallopess134@gmail.com; Lidia Beloni Silva: lidiabelonisilva@gmail.com; Claudia Bernardi Cesarino: claudiacesarino@famerp.br; Josimerci Ittavo Lamana Faria: ittavojosi@yahoo.com.br

\section{Resumo}

Objetivo: Verificar como o tema segurança do paciente está sendo desenvolvido em cursos de graduação em enfermagem. Material e métodos: Estudo descritivo, transversal e quantitativo, com participação de 167 estudantes do terceiro e quarto anos e 51 enfermeiros docentes de dois cursos (público e privado), por meio de questionário. Resultados: Constatou-se que em ambos, não há disciplina específica e maior enfoque está em conteúdos da área hospitalar, principalmente no processo de cuidar. A maioria acertou a definição da Organização Mundial de Saúde, afirmou que conhecia os passos para a segurança do paciente, mas errou a associação entre as ações preventivas de cada um, demonstrando divergências nas respostas, conhecimento insuficiente e erros conceituais. Conclusão: $O$ tema é desenvolvido de forma transversal, mas é necessária disciplina específica, com abordagem interdisciplinar para desenvolvimento de ações mais efetivas e eficazes no ensino, a fim de melhorar o conhecimento sobre segurança do paciente.

Palavras-chave: segurança do paciente, docentes, estudantes, curso de enfermagem.

\section{Abstract \\ Patient's safety: the theme development in nursing graduation courses}

A descriptive cross-sectional and quantitative study was carried out with 167 students and 51 teachers, through a structured questionnaire to observe how patient's safety is being developed in two Nursing undergraduate courses (public and private). No related school subject was found, and greater focus was pointed out on contents of the hospital environment. The majority of them could state on the definition of the World Health Organization, and had knowledge on the steps for patient's safety, but they made mistake on the association between preventive actions according to each of the 10 steps, demonstrating differences in answers, insufficient knowledge and conceptual errors. The need to have the inclusion of this specific school subject with an inter and transdisciplinary approach on patient's safety is important to develop more effective actions on care and teaching to improve nurses' training.

Key-words: Patient's safety, teachers, student, education nursing.

\section{Resumen}

Seguridad del paciente: desarrollo del tema en curso de pregrado en enfermería

Estudio descriptivo, transversal y cuantitativo, realizado con 167 estudiantes y 51 docentes, utilizando un cuestionario estructurado con el objetivo de verificar como el tema de seguridad del paciente es desarrollado en dos cursos de pregrado en enfermería (público y privado). Fue verificado que no hay disciplina específica, y es dado mayor enfoque en contenidos del área 
hospitalaria. La mayoría acertó la definición de la Organización Mundial de la Salud, afirmaron que conocían las etapas para la seguridad del paciente, pero se equivocó en la asociación entre las acciones preventivas de acuerdo con cada una de las 10 etapas, demostrando divergencias en las respuestas, conocimiento insuficiente y errores conceptuales. Hay necesidad de inclusión de la disciplina específica, con abordaje inter y transdisciplinar sobre seguridad del paciente para desarrollo de acciones más efectivas en la asistencia y en la enseñanza con la finalidad de mejorar la formación del enfermero.

Palabras-clave: seguridad del paciente, docentes, estudiantes, educación en enfermería.

Introdução

Em instituições hospitalares a segurança do paciente é preocupação constante. Os enfermeiros geralmente estão envolvidos com diagnósticos relacionados à identificação de anomalias que necessitam de intervenções e correções por parte da equipe multiprofissional que atua na assistência ao paciente. O diagnóstico, contendo percepções e atitudes dos profissionais, subsidia implementação de melhorias nos processos de trabalho [1].

A cultura da segurança deve ser o ponto de partida para as instituições de saúde [2]. Ela envolve política de qualidade implantada em cada serviço, com desenvolvimento de ações a fim de minimizar resultados desfavoráveis [3]. A segurança implica na redução de riscos de dano, com utilização de boas práticas assistenciais, baseadas em evidências científicas [4-6]. Desta maneira, é importante estabelecer uma cultura de comunicação de erros que favoreça discussão e aprendizado com os mesmos [7].

A notificação de erros, a busca e a análise dos fatores que o causaram dão subsídios para realizar ações corretivas a fim de reduzir os riscos relacionados à assistência em saúde. As instituições possuem normas a serem seguidas, que influenciam a maneira como os profissionais agem e interagem, de forma a colaborar com a cultura e o clima organizacional nos serviços [8]. Para implantação de ações preventivas, faz-se necessário esforço, persistência e desenvolvimento de práticas que possam conduzir mudanças de comportamento dos profissionais envolvidos na assistência [9].

A qualidade em serviços de saúde envolve gestão estratégica, visão, missão e valores da instituição. As metas e objetivos devem estar claros, a fim de beneficiar os pacientes no contexto do atendimento, por meio de protocolos assistenciais, equipe multiprofissional qualificada e comprometida com a cultura da segurança, efetividade, eficiência, segurança, inovação e tecnologia $[10,11]$. Entre as estratégias realizadas com a participação do Ministério de Saúde, em algumas ações globais estabelecidas pela Aliança Mundial para a Segurança do Paciente, vinculada à Organização Mundial da Saúde, destacou-se como primeiro desafio global: "Uma assistência limpa é uma assistência segura" e segundo: "Cirurgias seguras salvam vidas", priorizando a identificação e redução de eventos adversos evitáveis nos serviços de saúde [12].

O Programa Nacional de Segurança do Paciente (PNSP), criado em 2013, fomenta a inclusão do tema segurança do paciente em cursos técnicos, de graduação e pós-graduação na saúde [13]. A Rede Brasileira de Enfermagem e Segurança do Paciente (REBRAENSP), seguindo as recomendações da Rede Internacional de Enfermagem e Segurança do Paciente (RIENSP) objetiva melhorar as práticas de segurança do paciente, unindo esforços em nível mundial para prestação do cuidado seguro [14]. O trabalho em rede está sendo difundido por meio de polos e núcleos regionais, que compartilham ações sobre segurança do paciente, assim como incentivam a criação de Comitês de Segurança nos serviços de saúde.

Em instituições de saúde, os profissionais da educação permanente e gerência de risco reforçam os conhecimentos acerca das práticas seguras e dos riscos que envolvem a qualidade e segurança do paciente por meio de treinamentos sobre protocolos assistenciais $e$ ações voltadas à segurança do paciente [15]. A complexidade e alta tecnologia dos serviços de saúde exige mudança de comportamento da enfermagem, implicando em mudanças na formação do enfermeiro [16].

Os docentes exercem papel importante na definição de conceitos sobre qualidade e segurança do paciente, desenvolvimento de competências e habilidades para uma prática segura, centrada no paciente [17]. Nesta perspectiva, os cursos de graduação em enfermagem devem se envolver com conteúdos e práticas relacionadas à segurança durante a formação do enfermeiro, para que ele desenvolva uma cultura para segurança, com conhecimentos e habilidades para prevenir o erro humano [18]. 
O ensino de enfermagem vem se modificando ao longo do tempo, buscando formar profissionais com o desafio de prestar uma assistência de qualidade exigida pelo mundo atual, por meio de conhecimento científico, tecnológico e inovador [19]. Debates acerca das diretrizes curriculares para integrar o ensino ao serviço de saúde e estimular ações conjuntas, a partir da inserção crítica e responsável do profissional, vêm sendo estimulados nos últimos anos [7].

Almeja-se que os estudantes de graduação em enfermagem sejam os futuros líderes do cuidado, sendo necessário desenvolvimento de habilidades na aplicação de princípios e conceitos sobre segurança e práticas seguras. Portanto, a questão norteadora deste estudo foi: Como os cursos de graduação em enfermagem estão desenvolvendo o tema segurança do paciente? Diante disto, objetivou-se verificar como o tema segurança do paciente está sendo desenvolvido em cursos de graduação em enfermagem.

Material e métodos

Estudo descritivo, transversal e quantitativo, realizado com 51 enfermeiros docentes e 167 estudantes de dois cursos de graduação em enfermagem (um público e outro privado, denominados $\mathrm{A}$ e $\mathrm{B}$, respectivamente), em uma cidade do noroeste paulista, no período de agosto a dezembro de 2015.

Os critérios de inclusão para os estudantes eram estar cursando o terceiro ou quarto ano do curso de enfermagem e assinar o termo de consentimento. Foram excluídos o primeiro e segundo anos, por não terem cursado disciplinas que envolvem os cuidados diretos e indiretos ao paciente. Para os docentes foram ser enfermeiro, ministrar aulas no curso de graduação em enfermagem e assinar o termo de consentimento livre e esclarecido.

A instituição A é pública tinha 85 estudantes do terceiro e quarto anos, sendo 39 do terceiro, $35(89,74 \%)$ responderam ao questionário e 46 do quarto, e todos $(100 \%)$ participaram, totalizando uma amostra de 81estudantes (95,5\%). A instituição B é privada, tinha 90 estudantes do terceiro e quarto anos, 38 do terceiro, 37 (97,36\%) responderam ao questionário e 52 no quarto, $49(94,23 \%)$ participaram, totalizando uma amostra de 86 estudantes $(95,5 \%)$. A amostra foi correspondente ao número de estudantes dos dois cursos $(95,5 \%)$.

Para os docentes, foi utilizado um questionário dividido em duas partes: a primeira caracterizou os sujeitos; sexo, idade, instituição a qual pertencia, formação, instituição que se formou, tempo de formado, função que exercia na instituição e se possuía função assistencial em outra instituição, tempo de atuação como docente e atividades desenvolvidas no curso de graduação que atuava. A segunda parte continha 17 questões relacionadas ao conceito de segurança do paciente, disciplinas que contemplavam este conteúdo, estratégias de ensinoaprendizagem, experiência sobre evento adverso (EA) durante a vida acadêmica e sobre 0 conhecimento dos 10 passos para a segurança do paciente de acordo com as orientações contidas na cartilha do COREn de 2010 [16]. O questionário foi entregue em mãos pelo pesquisador no próprio ambiente de trabalho do participante.

O questionário utilizado pelos estudantes foi dividido em duas partes: a primeira caracterizou os sujeitos e a segunda parte continha 17 questões relacionadas à definição de segurança do paciente, disciplinas que contemplavam este conteúdo, estratégias de ensinoaprendizagem, período do primeiro contato com paciente, se receberam suporte teórico e laboratorial sobre a temática, se presenciaram algum EA durante as disciplinas e sobre 0 conhecimento dos 10 passos para a segurança do paciente descritos na cartilha do Coren de 2010 [16]. Os questionários foram entregues durante o intervalo das aulas dos respectivos cursos.

Foi realizada validação do questionário por três enfermeiros docentes que participavam do Núcleo de Segurança do Paciente de São José do Rio Preto e três alunos que não participaram como sujeitos da pesquisa. Essas pessoas atuaram como juízes, avaliaram a forma e o conteúdo do questionário e solicitaram modificação em duas questões relacionadas à definição da segurança do paciente, que não estavam claras, o que foi acatado pela pesquisadora.

Quanto à estatística, a análise inferencial das variáveis quantitativas foi realizada utilizando o Teste $T$ de Student. As comparações de frequências, envolvendo as variáveis qualitativas nominais foram realizadas por Teste Qui-quadrado, considerando $p \leq 0,05$ significativo, em ambos os testes. O programa utilizado foi o Graphpad Prism 6.01. O estudo foi aprovado pelo Comitê de Ética da Faculdade de Medicina de São José do Rio Preto- FAMERP. Parecer 168.753. 
Quanto à caracterização dos docentes, no curso A, a maioria se formou em instituição pública, todas eram mulheres, com idade entre 50 e 60 anos. No B, a maioria estudou em instituição privada, do sexo feminino, com idade entre 30 e 40 anos. A diferença de idade entre os docentes dos cursos foi significativa, com valor $p<0,0001$, os docentes do curso público apresentaram maior idade em relação ao privado.

No curso A, a maioria tem formação há mais de 20 anos, trabalham no curso em torno de 10 a 20 anos. No B, a maioria é formada há cerca de 5 a 10 anos, atuam no curso no período de 5 a 10 anos. A diferença entre os docentes quanto ao tempo de formado e atuação profissional como docente foi significativa, com valor de $p<0,0022$ e $p<0,0001$ respectivamente, sendo os docentes mais jovens pertencentes à instituição privada.

Em relação aos estudantes: no curso $A$, a maioria era do sexo feminino, com idade entre 20 e 25 anos no terceiro e quarto anos. No curso B, a maioria era do sexo feminino, com idade entre 20 - 25 anos e em torno de $30 \%$ acima de 30 anos. A diferença de idade entre os estudantes dos cursos foi significativa, com valor de $p<0.0001$. Na instituição pública os estudantes eram mais jovens.

Na instituição A, apenas 06 (7,50\%) trabalhavam na enfermagem e na B, 32 (37,64\%) trabalhavam na área, principalmente em hospitais, $20 \%$ são técnicos em enfermagem. A diferença entre os estudantes que trabalhavam e não trabalhavam, entre os dois cursos, foi significativa, com valor de $\mathrm{p}<0.0001$.

No que se refere à definição do tema segurança do paciente pelos docentes, no curso A, 17 (42,5\%) responderam correto, 21 (52,50\%) disseram que conheciam os 10 passos para a segurança, porém, mais de $50 \%$ deles não souberam associar as ações preventivas de cada um deles. No curso B, sete (63,63\%) acertaram a resposta, seis $(54,54 \%)$ responderam que conheciam os 10 passos, porém, quando comparadas às medidas de segurança de cada passo, houve incongruência nas respostas, pois mais de $80 \%$ não souberam associar as ações preventivas de cada um deles, como observado na Tabela I.

Tabela I - Demonstrativo do conhecimento dos docentes dos cursos $A$ e $B$, acerca da definição e dos 10 passos para segurança do paciente. São José do Rio Preto, 2015.

\begin{tabular}{llll}
\hline Variáveis & $\begin{array}{l}\text { Curso A } \\
\text { n (\%) }\end{array}$ & $\begin{array}{l}\text { Curso B } \\
\text { n (\%) }\end{array}$ & $\mathbf{p}$ \\
\hline $\begin{array}{l}\text { Definição } \\
\text { Respostas corretas }\end{array}$ & $17(42,50)$ & $07(63,63)$ & $\mathbf{0 . 3 7 0 7}$ \\
Respostas erradas & $23(57,50)$ & $04(36,36)$ & \\
Conhecimento dos 10 passos & & & 0.5363 \\
Sim & $21(52,50)$ & $06(54,54)$ & $05(45,45)$ \\
Não & $15(37,50)$ & - \\
Não responderam & $04(10,00)$ & \\
Medidas relacionadas aos 10 passos & & $09(81,81)$ & $\mathbf{0 . 1 3 7 7}$ \\
Todos os 10 passos & $19(47,50)$ & $01(9,09)$ \\
1. Identificação do paciente & $18(45,00)$ & $02(18,18)$ \\
2. Cuidado limpo e cuidado seguro, higienização & $18(45,00)$ & \\
das mãos & & $02(18,18)$ \\
3. Cateteres e sondas & $07(17,50)$ & $02(18,18)$ \\
4. Cirurgia segura & $16(40,00)$ & $02(18,18)$ \\
5. Administração segura de sangue e & $13(32,50)$ & \\
hemocomponentes & & - \\
6. Paciente envolvido com a sua própria & $09(22,50)$ & \\
segurança & & - \\
7. Comunicação efetiva & $08(20,00)$ & $01(9,09)$ \\
8. Quedas & $14(35,00)$ & $01(9,09)$ \\
9. Úlcera por pressão & $19(47,50)$ & $02(18,18)$ \\
10. Segurança no uso de tecnologias & $11(27,50)$ & \\
\hline
\end{tabular}

Os estudantes dos dois cursos apresentaram conhecimentos divergentes em relação à definição do tema. No curso A, do terceiro ano, apenas sete (20\%) acertaram a definição e os 
do quarto, apenas $13(28,88 \%)$. No B, 12 (36,36\%) do terceiro e apenas cinco (10,41\%) do quarto ano respondeu correto. A diferença de acertos entre os estudantes dos dois cursos foi significante, com valor $p<0.0164$, sendo maior número de acertos no curso $A$.

No curso $A$, do terceiro ano apenas um (2,85\%) trabalhava na área de enfermagem e acertou a questão e no quarto, cinco (10,86\%) trabalhavam e apenas um (25\%) acertou. No curso $B$, no terceiro ano $14(38,88 \%)$ trabalhavam na área, destes, apenas seis $(42,85 \%)$ acertaram a resposta e no quarto ano $18(37,50 \%)$ trabalhavam e apenas dois $(11,76 \%)$ acertaram.

Sobre os 10 passos para a segurança do paciente, os estudantes do curso $A$, do terceiro ano, $16(45,71 \%)$ e do quarto $25(54,34 \%)$ disseram que conheciam e no $\mathrm{B}$, do terceiro ano $17(47,22 \%)$ e do quarto $16(32,65 \%)$ conheciam, porém, quando comparado com as respostas das medidas de segurança, houve incongruência, pois em ambas as instituições, mais de $60 \%$ não souberam associar as ações preventivas corretas de cada um dos 10 passos para segurança do paciente, como observado na Tabela II.

Tabela II - Demonstrativo do conhecimento dos estudantes dos cursos $A$ e $B$ acerca da definição e dos 10 passos para segurança do paciente. São José do Rio Preto, 2015.

\begin{tabular}{|c|c|c|c|c|c|c|}
\hline \multirow[b]{2}{*}{$\begin{array}{l}10 \text { passos para a } \\
\text { segurança do } \\
\text { paciente }\end{array}$} & & \multicolumn{2}{|l|}{ Curso A } & \multicolumn{2}{|l|}{ Curso B } & \multirow[t]{2}{*}{$\begin{array}{l}\text { Valor } \\
\text { p }\end{array}$} \\
\hline & & $\begin{array}{l}\text { 3o } \\
\text { n (\%) }\end{array}$ & $\begin{array}{l}4^{\circ} \\
\text { n (\%) }\end{array}$ & $\begin{array}{l}\text { 3o } \\
\text { n (\%) }\end{array}$ & $\begin{array}{l}4^{\circ} \\
\text { n (\%) }\end{array}$ & \\
\hline \multirow{9}{*}{ Definição } & $\begin{array}{l}\text { Respostas } \\
\text { corretas }\end{array}$ & $\begin{array}{l}07 \\
(20,00)\end{array}$ & $13(28,88)$ & $11(33,33)$ & $05(10,41)$ & \multirow{4}{*}{0,0215} \\
\hline & Respostas erradas & $28(80)$ & $32(71,11)$ & $22(66,66)$ & $43(89,58)$ & \\
\hline & Não responderam & - & $01(2,17)$ & $04(10,81)$ & $01(2,04)$ & \\
\hline & Sim & $16(45,71)$ & $25(54,34)$ & $17(47,22)$ & $16(32,65)$ & \\
\hline & $\begin{array}{l}\text { Não } \\
\text { Não responderam }\end{array}$ & $19(54,28)$ & $21(45,65)$ & $\begin{array}{l}19(52,77) \\
01(2,70)\end{array}$ & $33(67,34)$ & \multirow[t]{6}{*}{0,2184} \\
\hline & $\begin{array}{l}\text { Identificação do } \\
\text { paciente } \\
\text { Cuidado limpo e }\end{array}$ & $24(68,57)$ & $26(56,52)$ & $18(48,65)$ & $22(44,90)$ & \\
\hline & $\begin{array}{l}\text { cuidado seguro, } \\
\text { higienização das } \\
\text { mãos }\end{array}$ & $24(68,57)$ & $28(60,87)$ & $21(56,76)$ & $26(53,06)$ & \\
\hline & $\begin{array}{l}\text { Cateteres e } \\
\text { sondas }\end{array}$ & $13(37,14)$ & $13(28,26)$ & $9(24,82)$ & $7(14,29)$ & \\
\hline & Cirurgia segura & $24(68,57)$ & $22(47,82)$ & $20(54,05)$ & $17(34,69)$ & \\
\hline \multirow[t]{6}{*}{ Conhecimento } & Administração & & & & & \\
\hline & $\begin{array}{l}\text { segura de sangue } \\
\text { e } \\
\text { hemocomponentes }\end{array}$ & $20(57,14)$ & $28(60,87)$ & $13(35,14)$ & $18(36,73)$ & \multirow[t]{5}{*}{0,9325} \\
\hline & $\begin{array}{l}\text { Paciente envolvido } \\
\text { com a sua própria } \\
\text { segurança }\end{array}$ & $17(48,57)$ & $21(45,65)$ & $9(24,82)$ & $19(38,78)$ & \\
\hline & $\begin{array}{l}\text { Comunicação } \\
\text { efetiva }\end{array}$ & $07(20,00)$ & $10(21,74)$ & $08(21,62)$ & $17(34,69)$ & \\
\hline & $\begin{array}{l}\text { Quedas } \\
\text { Úlcera por pressão }\end{array}$ & $\begin{array}{l}24(68,57) \\
25(71,43)\end{array}$ & $\begin{array}{l}26(56,52) \\
28(60,87)\end{array}$ & $\begin{array}{l}16(43,24) \\
19(51,35)\end{array}$ & $\begin{array}{l}28(57,14) \\
28(57,14)\end{array}$ & \\
\hline & $\begin{array}{l}\text { 10. Segurança no } \\
\text { uso de tecnologias }\end{array}$ & $05(14,29)$ & $05(10,87)$ & $08(21,62)$ & $09(18,37)$ & \\
\hline
\end{tabular}

Quanto à percepção dos docentes sobre disciplinas que abordam o conteúdo segurança do paciente, ficou destacado nos dois cursos: cuidar em enfermagem (Semiologia e Semiotécnica) a que mais contempla a temática, seguida da Saúde do Adulto e do Idoso. Entretanto, no curso privado mais de $90 \%$ disseram que o Estágio Supervisionado, realizado no último ano do curso é o que mais aborda. 
As estratégias de ensino-aprendizagem utilizadas no desenvolvimento de conteúdo sobre segurança do paciente mais citadas pelos docentes do curso A foram: discussão de casos $32(80 \%)$ e aulas expositivas 27 (67,50\%). Enquanto no B, $11(100 \%)$ relataram aulas expositivas e realização de discussão de casos, oito $(72,72 \%)$ também citaram laboratórios de aprendizagem. Quando questionados sobre terem presenciado evento adverso (EA) durante a vida profissional, $32(80 \%)$ da instituição A disseram que passaram por essa experiência, e na B 10 (90,90\%). Entretanto, a maioria não causou nenhum dano ao paciente.

Tabela III - Disciplinas que abordam conteúdo sobre segurança do paciente, estratégias utilizadas e eventos adversos na percepção dos docentes dos cursos $A$ e B. São José do Rio Preto, 2015.

\begin{tabular}{llll}
\hline Variáveis & $\mathbf{A}$ & $\mathbf{B}$ & $\mathbf{p}$ \\
& $\mathbf{n}(\%)$ & $\mathbf{n}(\%)$ & \\
\hline Disciplinas & & & \\
Cuidar em enfermagem & $36(90,00)$ & $09(81,81)$ & 0.9646 \\
Saúde do adulto & $32(80,00)$ & $09(81,81)$ & \\
Saúde da criança & $28(70,00)$ & $07(63,63)$ & \\
Saúde da mulher & $27(67,50)$ & $07(63,63)$ & \\
Saúde coletiva & $27(67,50)$ & $08(72,72)$ & \\
Saúde do homem & $25(62,50)$ & $09(81,81)$ & \\
Saúde mental & $26(65,00)$ & $08(72,72)$ & \\
Estágio Supervisionado & $30(75,00)$ & $10(90,90)$ & \\
Outras & $06(15,00)$ & $04(36,36)$ & \\
Estratégias utilizadas & & & \\
Aulas expositivas & $27(67,50)$ & $11(100)$ & 0.8561 \\
Discussão de casos & $32(80,00)$ & $11(100)$ & \\
Aulas em laboratórios com manequins & $17(42,50)$ & $08(72,72)$ & \\
Aulas com simulação em computadores & $03(7,50)$ & $03(27,27)$ & \\
Educação à distância & $03(7,50)$ & $02(18,18)$ & \\
Outras & $06(15,00)$ & $02(18,18)$ & \\
Presenciou algum evento adverso & & & \\
Sim & $32(80,00)$ & $10(90,90)$ & 0.0008 \\
Não & $04(10,00)$ & $01(9,09)$ & \\
Não responderam & $04(10,00)$ & - & \\
Causou algum dano durante a sua vida profissional & & & \\
Sim & $07(17,50)$ & $01(9,09)$ & 0.5678 \\
Não & $31(77,50)$ & $10(90,90)$ & \\
Não responderam & $02(5,00)$ & - & \\
\hline
\end{tabular}

A maioria dos estudantes do curso A, 17 (48,57\%) do terceiro e 27 (58,69\%) do quarto ano, afirmou que as melhores estratégias de ensino-aprendizagem são aulas expositivas e laboratórios. Na B a mais citada pelos alunos do terceiro ano, 15 (40,54\%) foi aula expositiva e do quarto, $16(32,65 \%)$ prática em laboratórios.

Quando questionados sobre ter presenciado EA durante a graduação, houve diferença significativa entre os estudantes dos dois cursos, com valor $p<0.0001$, do terceiro ano 24 $(58,57 \%)$ e do quarto $36(78,26 \%)$ da instituição A relataram ter vivido essa experiência, enquanto na $B$, apenas oito $(21,62 \%)$ do terceiro e $12(24,48 \%)$ do quarto ano.

A realização de prática clínica pelos estudantes de ambos os cursos ocorreu durante 0 terceiro período do curso de graduação. Quanto ao suporte teórico e prático-laboratorial antes do primeiro contato com paciente em instituições de saúde, $141(84,43 \%)$ relataram ter recebido. Porém, essa descrição é significativa, com valor $p<0.0001$, os estudantes do curso privado relataram ter recebido maior conteúdo sobre segurança do paciente.

Sobre os estudantes sentirem-se seguros na realização de procedimentos assistenciais de enfermagem houve diferença significativa entre os dois cursos, com valor $p<0.0001$. Dos estudantes do curso A, $82 \%$ relataram que se sentem seguros, e no B, $67 \%$. 
O gênero feminino predominou entre os docentes e estudantes. A quantidade de homens cursando enfermagem foi menor que $6 \%$ nos dois cursos, o que pode ser justificado pela história da profissão e o fato da enfermagem ser exercida por mulheres tanto na assistência como no ensino [18,20,21]. Quanto à docência, a maioria do curso $A$ possuía dedicação exclusiva, e no $B$ a metade deles possuía outro emprego concomitante. A docência é uma das funções do enfermeiro, portanto, é necessário investir na formação e capacitação do enfermeiro para o ensino, com uma visão geral de educação [22].

A diferença de idade entre os docentes e estudantes dos dois cursos foi significativa. A instituição pública possui mais jovens em relação à privada. Corroborando estudos realizados também em instituições privadas, em que os estudantes tinham idade acima de 30 anos $(31,60 \%)$ [23] e nas públicas a maioria dos estudantes é jovem, entre 20 a 30 anos [24].

Nesta pesquisa, nos dois cursos, mais da metade dos docentes relataram conhecer os 10 passos para a segurança do paciente. Entretanto, aproximadamente 50\% erraram a definição e mais de $50 \%$ deles não souberam associar as ações preventivas de cada um dos passos. Quanto aos estudantes, mais da metade relatou conhecer os 10 passos para a segurança do paciente, porém, aproximadamente $60 \%$ não souberam correlacionar às definições com as ações de cada item e as práticas que devem ser desenvolvidas. Em 2010, baseado nas seis metas internacionais para segurança do paciente, o Conselho Regional de Enfermagem (COREn) [16] lançou a cartilha com os 10 passos para a segurança do paciente, distribuídas aos enfermeiros, com ampla repercussão na enfermagem, portanto esperava-se maior conhecimento e acertos dos docentes e estudantes sobre essa temática.

$\mathrm{Na}$ grade curricular dos dois cursos não há disciplina específica sobre segurança do paciente. Em ambos, o tema estava inserido dentro das disciplinas, de maneira transversal. $\mathrm{O}$ Programa Nacional de Segurança do Paciente juntamente com o Ministério da Saúde e com o Conselho Nacional de Educação sugerem a inclusão do tema segurança como disciplina nos currículos dos cursos da área da saúde [13].

O Guia Curriculum de Segurança do Paciente da OMS, criado em 2011 é um programa que ajuda na implantação da educação para a segurança do paciente em instituições de ensino de todo o mundo. Também sugere alguns métodos de ensino para se introduzir o tema em currículos existentes, incluindo simulação, palestras interativas e didáticas, grupos de discussão e práticas clínicas. Embora existam barreiras a serem superadas ao se adicionar disciplina específica sobre segurança do paciente, reconhece-se a importância para a formação do enfermeiro, com o envolvimento de docentes e estudantes neste contexto [12].

A abordagem do tema segurança do paciente em currículo de cursos de graduação é mais frequente em países desenvolvidos, como Estados Unidos da América (EUA) e Reino Unido, em geral, como disciplina optativa, não havendo inclusão formal obrigatória na estrutura curricular educacional em saúde [14]. No Japão, estudo realizado com 83 instituições de ensino em enfermagem, sendo $55 \%$ públicas e $45 \%$ privadas constatou que elas utilizam cerca de nove a 14 horas de conteúdo relacionado à segurança do paciente, respectivamente [25].

No contexto de uma formação crítica-reflexiva com enfoque para a realidade da saúde apontam-se a importância no trabalho docente enquanto mediador do processo ensinoaprendizagem, a partir da elaboração de estratégias de ensino em sala de aula, com a finalidade de formar profissionais com atitude de aprender a aprender. O docente tem uma atuação estratégica, pois exerce o papel de traduzir a ideia principal para o contexto da prática [26].

As estratégias de ensino utilizadas pelos docentes foram aulas expositivas e a maioria dos alunos avaliou como boa. Estudos mostram algumas estratégias utilizadas para estimular o pensamento crítico-reflexivo dos estudantes como: simulação em laboratório, questionamento, estudo de caso, ensino online e aprendizagem interativa, mapa conceitual e aprendizagem baseada em problemas [27,28].

Estudo realizado no Iran no ano de 2015 ressalta a necessidade de estudantes e profissionais da saúde se familiarizarem com barreiras que previnem erros e utilização de melhores práticas assistenciais. Constatou-se que ainda há pouco conhecimento sobre os mecanismos multifatoriais que envolvem a ocorrência de erro, por isso o desenvolvimento de uma cultura para segurança do paciente nos currículos das profissões da área da saúde é essencial para a melhoria dos serviços [29].

Houve incongruência na resposta dos estudantes em relação ao sentimento de segurança para realização de procedimentos durante a graduação. A maioria errou as práticas 
relacionadas aos 10 passos para segurança do paciente do COREn [16]. Para implantação de ações preventivas, é necessário mudança de comportamento, esforço, persistência e desenvolvimento de práticas que possam conduzir às mudanças. Os profissionais de enfermagem são os que ficam maior tempo com o paciente, logo devem conhecer e aceitar os riscos que envolvem a sua profissão, o ambiente de trabalho, a equipe multiprofissional e estar consciente e envolvido com práticas seguras [30].

No processo ensino-aprendizagem, o uso da simulação é uma estratégia inovadora e complementar que deve ser incentivada. Estudo realizado com estudantes de $4^{\circ}$ e $5^{\circ}$ ano de graduação em enfermagem demonstrou que mais de $65 \%$ acreditam que a simulação em laboratório consolida o aprendizado, contribuindo para superação das dificuldades de assimilação e controle do estresse emocional, além de reforçar a interação entre professor e aluno [31].

A utilização de novas estratégias de ensino no processo de aprendizagem deve agregar valor à prática e transcender novos horizontes [32]. $\mathrm{Na}$ atualidade, a questão da segurança do paciente é um problema de saúde mundial [33]. Portanto, os profissionais de saúde, especialmente os enfermeiros, devem conhecer os 10 passos para a segurança do paciente e os processos de trabalho que envolve a qualidade e segurança nos serviços de saúde.

\section{Conclusão}

Quanto ao desenvolvimento de práticas seguras e do tema segurança do paciente, houve divergências nas respostas, demonstrando conhecimento insuficiente e erros conceituais tanto dos docentes como dos estudantes dos dois cursos de graduação em enfermagem. O tema é desenvolvido de forma transversal em ambos, no entanto é necessário disciplina específica, com abordagem interdisciplinar para implementação de ações mais efetivas e eficazes no ensino, a fim de melhorar o conhecimento e propiciar ao enfermeiro conscientização e envolvimento com a cultura de segurança do paciente.

Novas pesquisas a fim de elaborar estratégias adequadas para o desenvolvimento do tema segurança do paciente nos cursos de graduação em enfermagem se fazem necessárias, a fim de propiciar melhor formação profissional e consequentemente uma assistência mais segura.

1. Reis CT, Martins M, Laguardia J. A segurança do paciente como dimensão da qualidade do cuidado de saúde - um olhar sobre a literatura. Rev Ciênc Saúde Coletiva 2013;18(7):2029-36.

2. Sexton JB, Helmreich RL, Neilands TB, Rowan K, Vella K, Boyden J et al. The safety attitudes questionnaire: psychometric properties, benchmarking data, and emerging research. BMC Health Serv Res 2006;44(6):1-10.

3. Beattie M, Murphy DJ, Atherton I, Lauder Instruments to measure patient experience of healthcare quality in hospitals: a systematic review. Syst Rev 2015;4:97.

4. World Health Organization (WHO). More than words. Conceptual framework for the International Classification for Patient Safety (ICPS): technical report. Geneva: WHO; 2009.

5. Ministério da Saúde. Fundação Oswaldo Cruz. Agência Nacional de Vigilância Sanitária. Documento de referência para o programa nacional de segurança do paciente. Brasília: Ministério da Saúde; 2014.

6. Gouvêa CSD, Travassos C. Indicadores de segurança do paciente para hospitais de pacientes agudos: revisão sistemática. Cad Saúde Pública 2010;26(6):1061-78.

7. Urbanetto JS, Gerhardt LM. Segurança do paciente na tríade assistência ensino pesquisa [Editorial]. Rev Gaúcha Enferm 2013;34(3):8-9.

8. Tomazoni A, Rocha PK, de Souza S, Anders JC, Malfussi HFC. Cultura de segurança do paciente em unidades de terapia intensiva neonatal: perspectivas da equipe de enfermagem e médica. Rev Latinoam Enferm 2014;22(5):755-63.

9. Harada MJCS, Pedreira MLG. O erro humano e sua prevenção. In: Agência Nacional de Vigilância Sanitária. Assistência Segura: uma reflexão teórica aplicada à prática. Brasília: Agencia Nacional de Vigilância Sanitária; 2013. p.41-54. 
10. Fernandes HS, Júnior SAP, Costa RF. Qualidade em terapia intensiva. Rev Bras Clin Med 2010;(8):37-45.

11. Tase TH, Lourenção DCA, Bianchini SM, Tronchin DMR. Identificação do paciente nas organizações de saúde: uma reflexão emergente. Rev Gaúcha Enferm 2013;34(2):196200.

12. Bogarin DF, Zanetti AC, Brito MF, Machado JP, Gabriel CS, Bernardes A. Segurança do paciente: conhecimento de alunos de graduação em enfermagem. Cogitare Enferm 2014;19(3):491-7.

13. Brasil. Ministério da Saúde. Institui o Programa Nacional de Segurança do Paciente (PNSP). Portaria n.529, de 1 de abril de 2013. Art. 3, inciso V.

14. Rede Brasileira de Enfermagem e Segurança do Paciente. Estratégias para a segurança do paciente: manual para profissionais da saúde. Porto Alegre: EDIPUCRS; 2013.

15. Cararro TE, Gelbcke FL, Sebold LF, Kempfer SS, Zapelini MC, Waterkemper R. A biossegurança e segurança do paciente na visão de acadêmicos de enfermagem. Rev Gaúcha Enferm 2012;33(3):14-9.

16. Conselho Regional de Enfermagem do Estado de São Paulo (COREN-SP). Cartilha dos 10 passos da segurança do paciente. São Paulo: REBRAENSP; 2010.

17. Souza NVDO, Penna LHG, Cunha LS, Baptista AAS, Mafra IF, Mariano DCA. Perfil socioeconômico e cultural do estudante ingressante no curso de graduação em enfermagem. Rev Enferm UERJ 2013;21(2):718-22.

18. Nardelli GG, Gaudenci EM, Garcia BB, Carleto CT, Gontijo LM, Pedrosa LAK. Perfil dos alunos ingressantes dos cursos da área da saúde de uma universidade federal. Rev Enferm Atenção Saúde 2013;2(1):3-12.

19. Erdmann AL, Fernandes JD, Teixeira GA. Panorama da educação em enfermagem no Brasil: graduação e pós-graduação. Enfermagem em Foco 2011; 2(supl):89-93.

20. Hirsch CD, Barlem ELD, Duarte FD, Fornari NC, Silva BR, Nogário ACD. Sociodemographic and academic profile of undergraduate nursing student of the Nursing School/FURG. J Nurs Socioe Health 2014;1(1):13-8.

21. Freitas EO, Bublitz S, Neves ET, Guido LA. Sociodemographic and academic profile of nursing students of a public University. Rev Enferm UFPE 2012;6(10):2455-62.

22. Rocha ALF, Fonseca MG. Reflexões sobre a docência em enfermagem. As características necessárias ao enfermeiro professor segundo a literatura. EF Desportes 2012;17(174):1-6.

23. Corrêa AK, Souza MCBM, Santos RA, Clapis MJ, Granvile NC. Perfil de estudantes ingressantes em licenciatura: escola de enfermagem de Ribeirão Preto da Universidade de São Paulo. Rev Esc Enferm USP 2011;45(4):933-8.

24. Vall J, Pereira LF, Friesen TT. O perfil do acadêmico de enfermagem em uma faculdade privada da cidade de Curitiba. Cad Esc Saúde 2009;1(2):1-10.

25. Maeda S, Kamishiraki E, Starkey J, Ehara K. Patient safety education at Japanese nursing schools: results of a nationwide survey. BMC Res Notes 2011;4:416.

26. Cruz BM, Dvolatka JM, Baumel JA, Gaspar MDR, Pires SMB. O papel do enfermeiro docente na formação inicial do técnico de enfermagem na segurança do paciente. In: 4ํㅡㄹ Congresso Internacional de Educação, Pesquisa e Gestão; 2012; Ponta Grossa. Ponta Grossa: Instituto Sul Americano de Pós-Graduação, Ensino e Tecnologia; 2012.

27. Morelato CS, Camargo RAA. Estratégias de ensino na educação profissional de nível médio em enfermagem. In: 5ª Jornada das Licenciaturas da USP; 2014; São Carlos. São Carlos: Universidade de São Paulo; 2014.

28. Waterkemper R, Prado ML. Estratégias de ensino-aprendizagem em cursos de graduação em Enfermagem. Av Enferm 2011;29(2):234-46.

29. Martins JCA, Mazzo A, Baptista RCN, Coutinho VRD, Godoy S, Mendes IAC, et al. A experiência clínica simulada no ensino de enfermagem: retrospectiva histórica. Acta Paul Enferm 2012;25(4):619-25.

30. Belela-Anacleto ASC, Sousa BEC, Yoshikawa JM, Avelar AFM, Pedreira MLG. Higienização das mãos e a segurança do paciente: perspectiva de docentes e universitários. Texto Contexto - Enferm 2013;22(4):901-8.

31. Nabilou B, Feizi, Seyedin H.pacient safety in medical education: students' perceptions, knowledge and attitudes. PLoS ONE 10(8),2015. doi: 10.1371/journal.pone0135610 
32. Valadares AFM, Magro MCS. Opinião dos estudantes de enfermagem sobre a simulação realística e o estágio curricular em cenário hospitalar. Acta Paul Enferm 2014;27(2):138-43.

33. Montserrat-Capella D, Suárez M, Ortiz L, Mira JJ, Duarte HG, Reveiz L, et al. Frequency of ambulatory care adverse events in Latin American countries: the AMBEAS/PAHO cohort study. Int J Qual Health Care 2015;27(1):52-9. 